\title{
Analysis of psoriasis-relevant gene expression and exon usage changes upon the altered expression of splicing factors differentially expressed in psoriatic epidermis
}

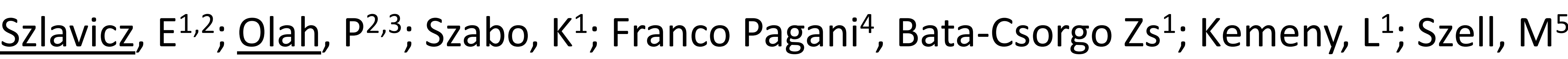 \\ ${ }^{1}$ Dept. of Dermatology and Allergology, Albert Szent-Györgyi Clinical Center, University of Szeged, Hungary \\ 2Dept. of Dermatology, Venerology and Oncodermatology, University of Pécs, Hungary \\ ${ }^{3}$ Dept. of Dermatology, Heinrich Heine University, Duesseldorf \\ ${ }^{4}$ International Centre for Genetic Engeneering and Biotechnology, Trieste, Italy \\ ${ }^{5}$ Dept. of Medical Genetics, Albert Szent-Györgyi Clinical Center, University of Szeged, Hungary
}
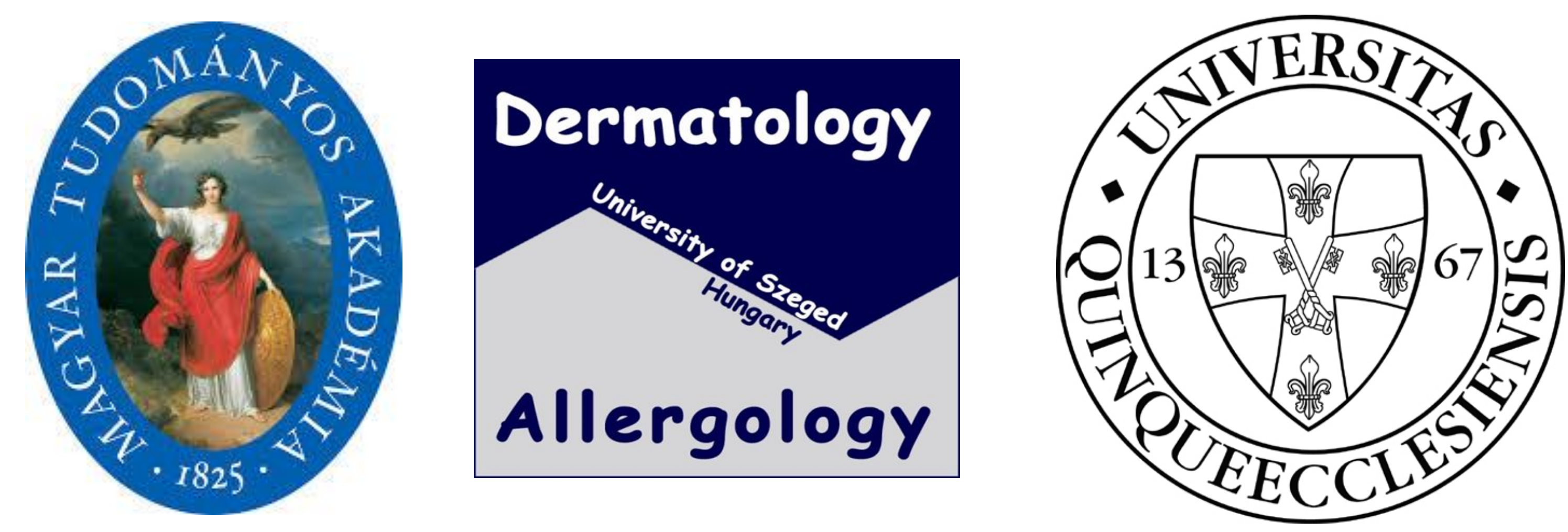

INTRODUCTION: LUC7L3, PPIG and SFSR18 SR-rich splicing factors have been previously identified to be differentially regulated in healthy and psoriatic non-involved epidermis, upon T-lymphokine stimulation. We have demonstrated their elevated levels both in non-lesional and lesional psoriatic epidermis samples and the combined silencing of LUC7L3/SFRS18 have been verified to be the most efficient in splicing pattern alteration of the psoriasis-associated fibronectin isoform, the EDA+ fibronectin.

AIMS: Comparison of distinct gene expression alterations and differential exon usage upon double silencing of LUC7L3 and SFRS18.

MATERIALS AND METHODS: cDNA 2x100bp paired-end RNA-sequencing libraries were prepared and sequenced on one lane of the Illumina HiScan SQ platform, resulting in $\sim 35 \mathrm{M}$ reads per sample sequencing depth. Raw reads were adapter and quality filtered using the FastQC and the fastx toolkits, followed by mapping with TopHat2 and isoform reconstruction in Cufflinks. Differential expression and differential exon usage were analyzed by DESeq and DEXSeq in R.

\section{RESULTS}

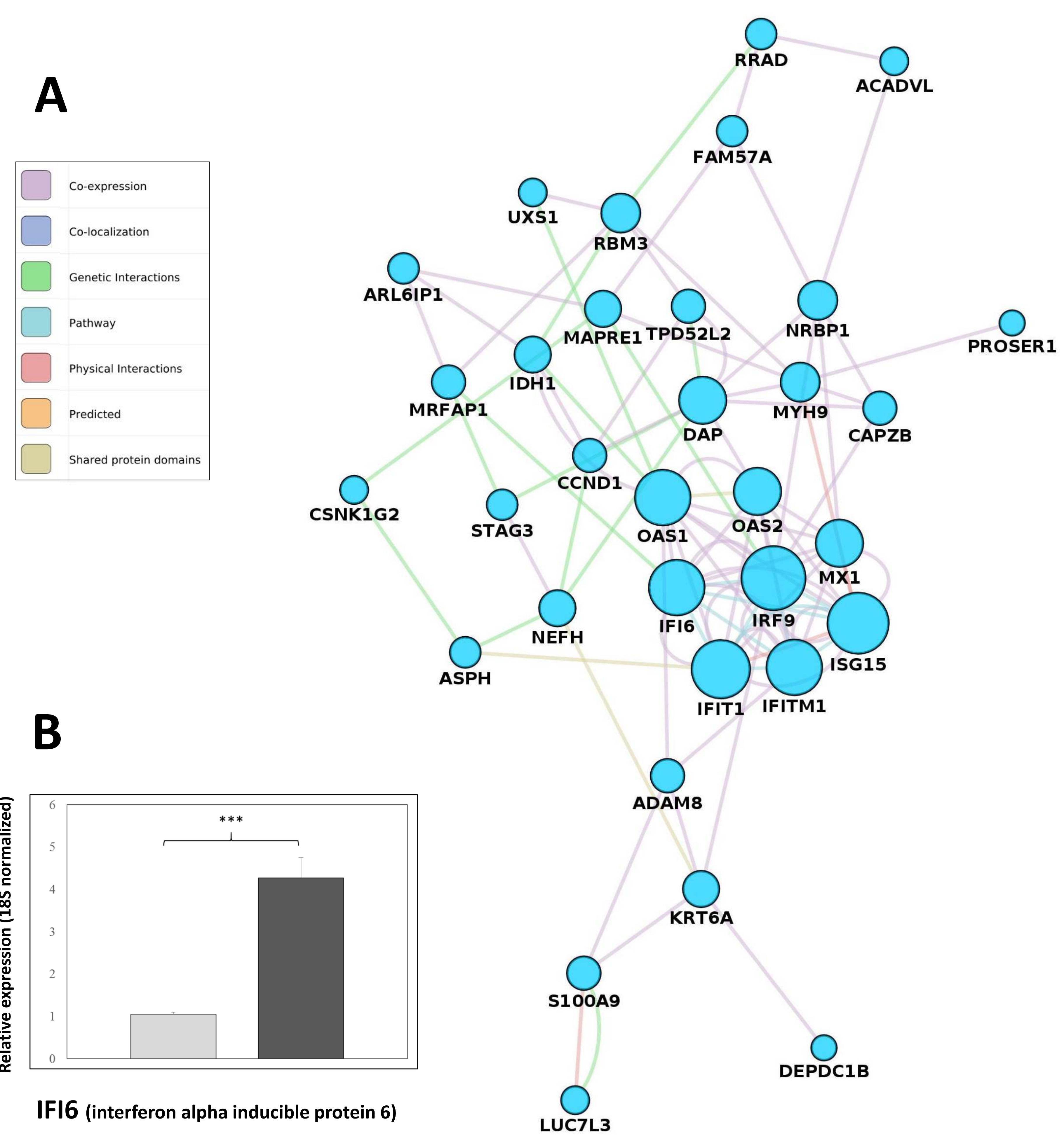

Fig.1.A: Differential gene expression: LUC7L3/SFRS18 combined silencing resulted in moderate, but significant changes in gene expression, accompanied by extensive disturbances in exon inclusion and exclusion rates. As a result, 35 genes were identified as differentially expressed $(\operatorname{logFC}>0.5$, FDR $<0.05)$, with IFI6 and MX1, ISG15 and KRT6A mRNAs showing the most robust fold-changes.

Fig.1.B: qRT-PCR validation of IFI6 expression indicated 4-fold changes $\left(\log _{2} \mathrm{FC}=2\right)$, in agreement with the RNASeq results.

CONCLUSION: LUC7L3 and SFRS18 splicing factors contribute to the regulation of several well-known psoriasis-associated pathways, including the IFN signaling pathway (IFI6, IFIT1, IFIT2, IRF9, ISG15), antiviral and innate immunity (S100A9, KRT6A, OAS1, OAS2, MX1), and ubiquitination (HERC6, CUL1). These results, together with our results for long non-coding RNA expression and exon usage changes, might open new insights to the molecular disturbances in early psoriasis development caused by altered mRNA maturation.
Fig.2: Differential exon usage was detected in 224 exons of 217 genes at $\log F C>0.5$, FDR $<0.1$, corresponding to $p$-value $<7 \times 10^{-4}$. Differential exon usage was identified in several non-coding RNAs (NEAT1, TINCR), whereas functional network analysis of the most significant genes (CREB1, CUL1, FN1, HERC6, NBEAL1), indicated an extensive co-expression background, suggesting overlapping regulation at the transcriptional and post-transcriptional levels.

Red indicates the average exon usage frequency in control samples, and blue in silenced samples. Significantly differentially expressed exons are indicated in pink.
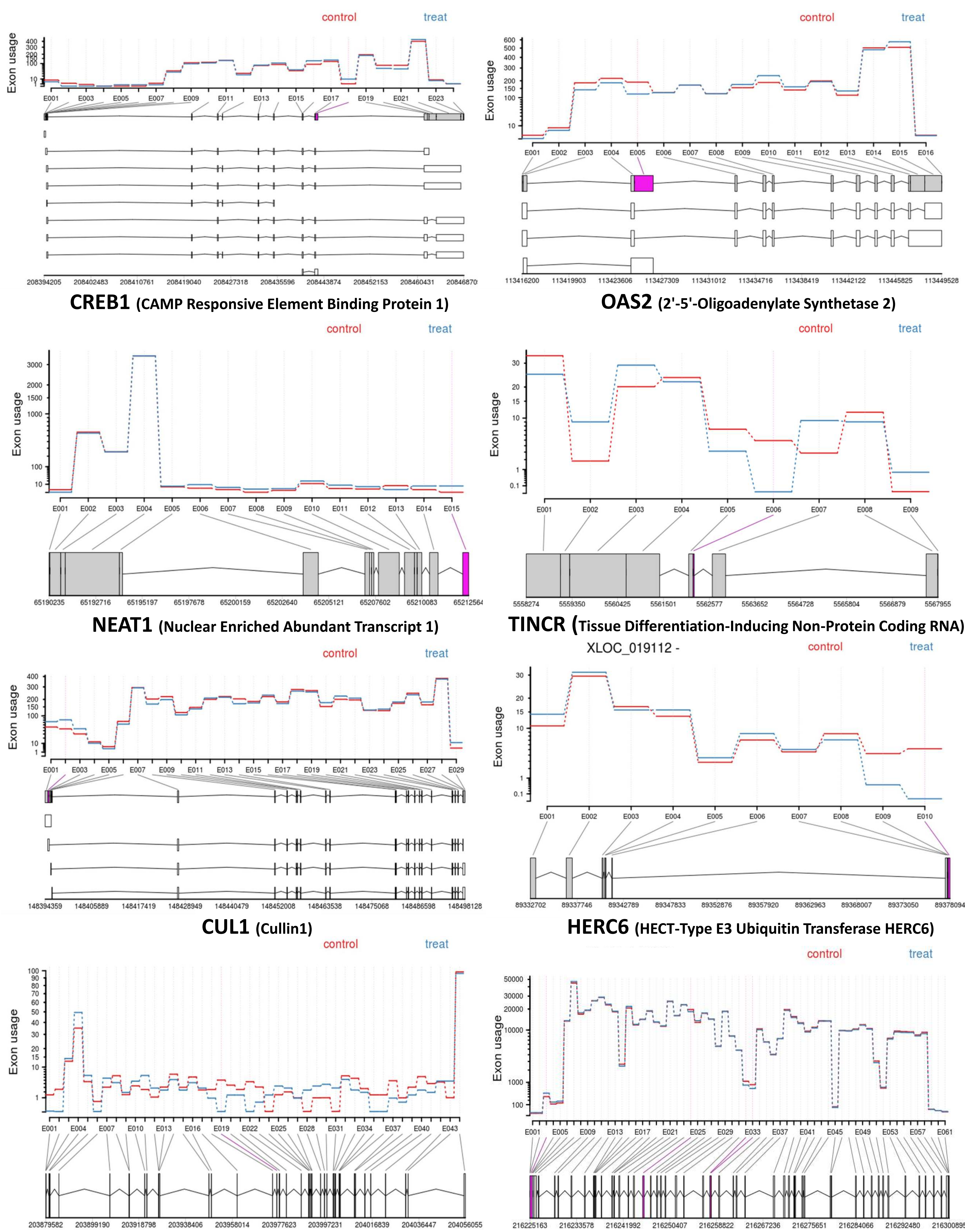

HERC6 (HECT-Type E3 Ubiquitin Transferase HERC6)

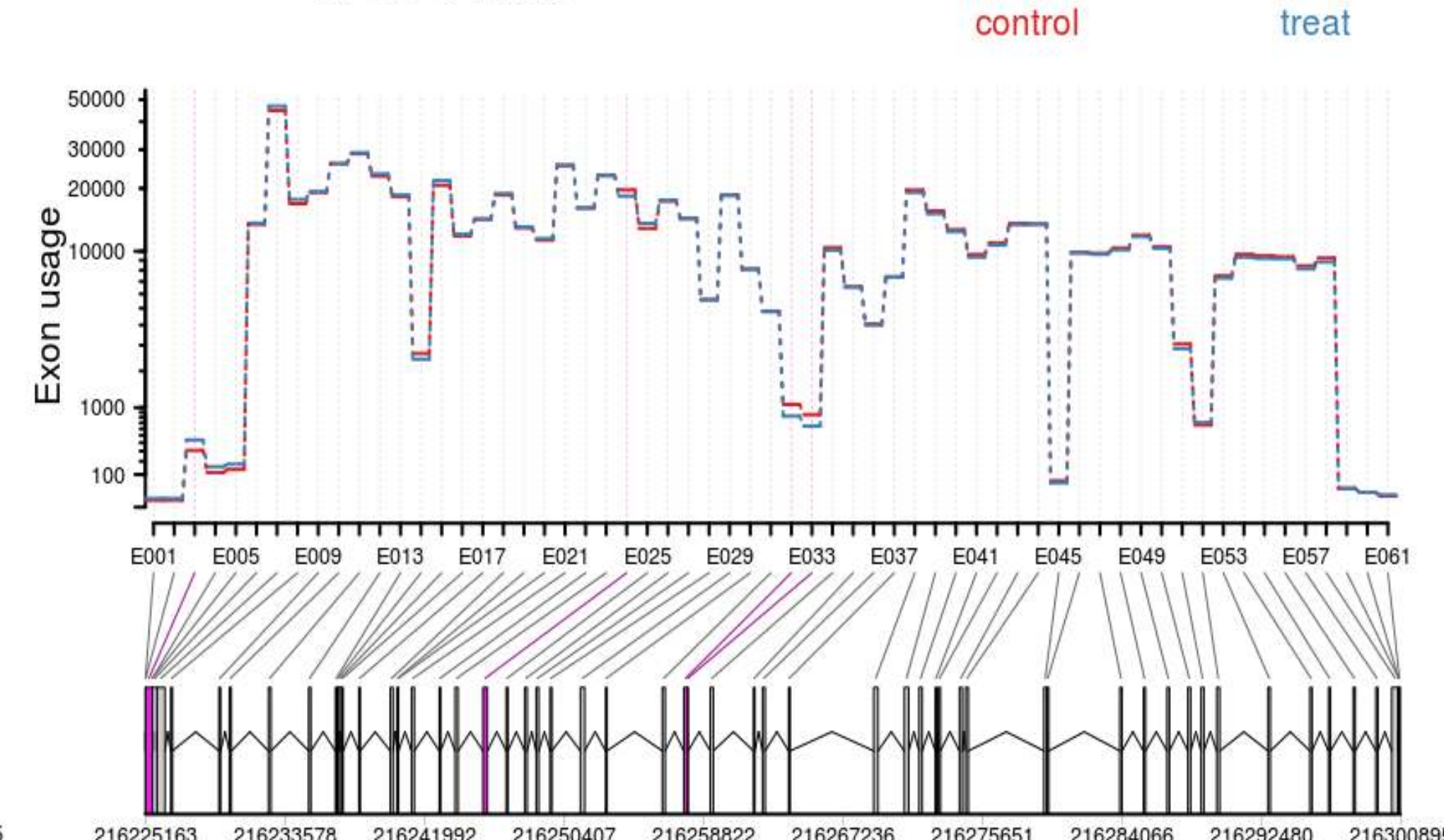

NBEAL1 (Neurobeachin Like 1)

FN1 (Fibronectin)

ACKNOWLEDGEMENT: The work was supported by OTKA grants $5 \mathrm{~K} 321$ and K105985, and by GINOP-2.3.2-15-2016-00015 and GINOP-2.2.1-15-2016-00007 research grants. 